\title{
Lambda means clustering: Automatic parameter search and distributed computing implementation
}

\section{Citation}

Comiter, Marcus, Miriam Cha, H. T. Kung, and Surat Teerapittayanon. 2016. "Lambda means clustering: Automatic parameter search and distributed computing implementation." In Proceedings of 23rd International Conference on Pattern Recognition (ICPR), Cancún, Mexico, December 4-8, 2016: 2331-2337.

\section{Published Version}

doi:10.1109/ICPR.2016.7899984

\section{Permanent link}

http://nrs.harvard.edu/urn-3:HUL.InstRepos:34390104

\section{Terms of Use}

This article was downloaded from Harvard University's DASH repository, and is made available under the terms and conditions applicable to Open Access Policy Articles, as set forth at http:// nrs.harvard.edu/urn-3:HUL.InstRepos:dash.current.terms-of-use\#OAP

\section{Share Your Story}

The Harvard community has made this article openly available.

Please share how this access benefits you. Submit a story.

\section{Accessibility}




\section{LAMBDA MEANS CLUSTERING AUTOMATIC PARAMETER SEARCH AND DISTRIBUTED COMPUTING IMPLEMENTATION}

MARCUS COMITER, MIRIAM CHA, HT KUNG, SURAT TEERAPITTAYANON HARVARD UNIVERSITY 
TALK OUTLINE

- Motivation and Introduction

- Background

- Lambda Means

- Benefits of Lambda Means

- Results

- Extension to Distributed Framework 
MACHINE LEARNING: VISION VS. REALITY 


\section{MACHINE LEARNING: VISION VS. REALITY}

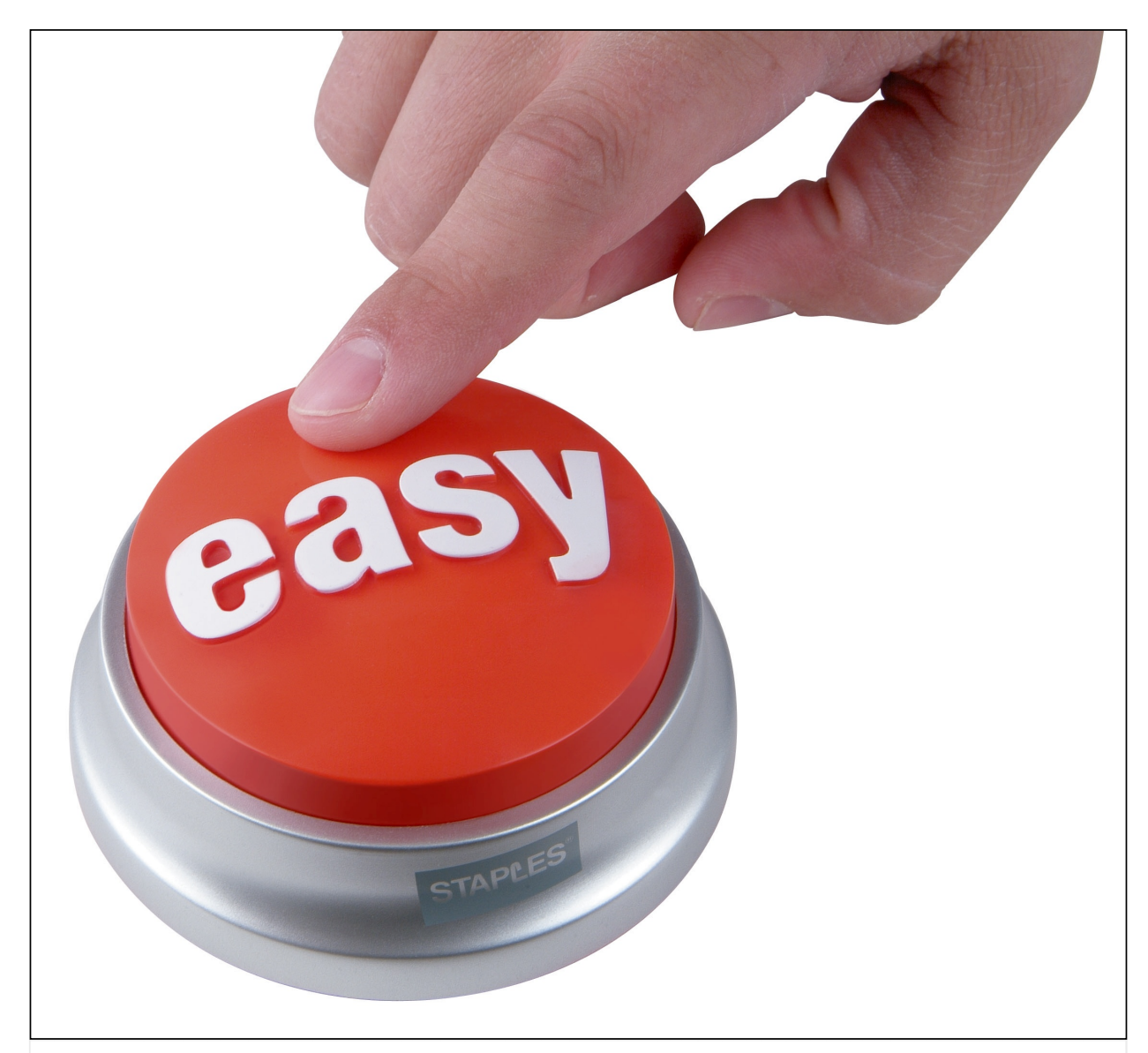

Vision 


\section{MACHINE LEARNING: VISION VS. REALITY}

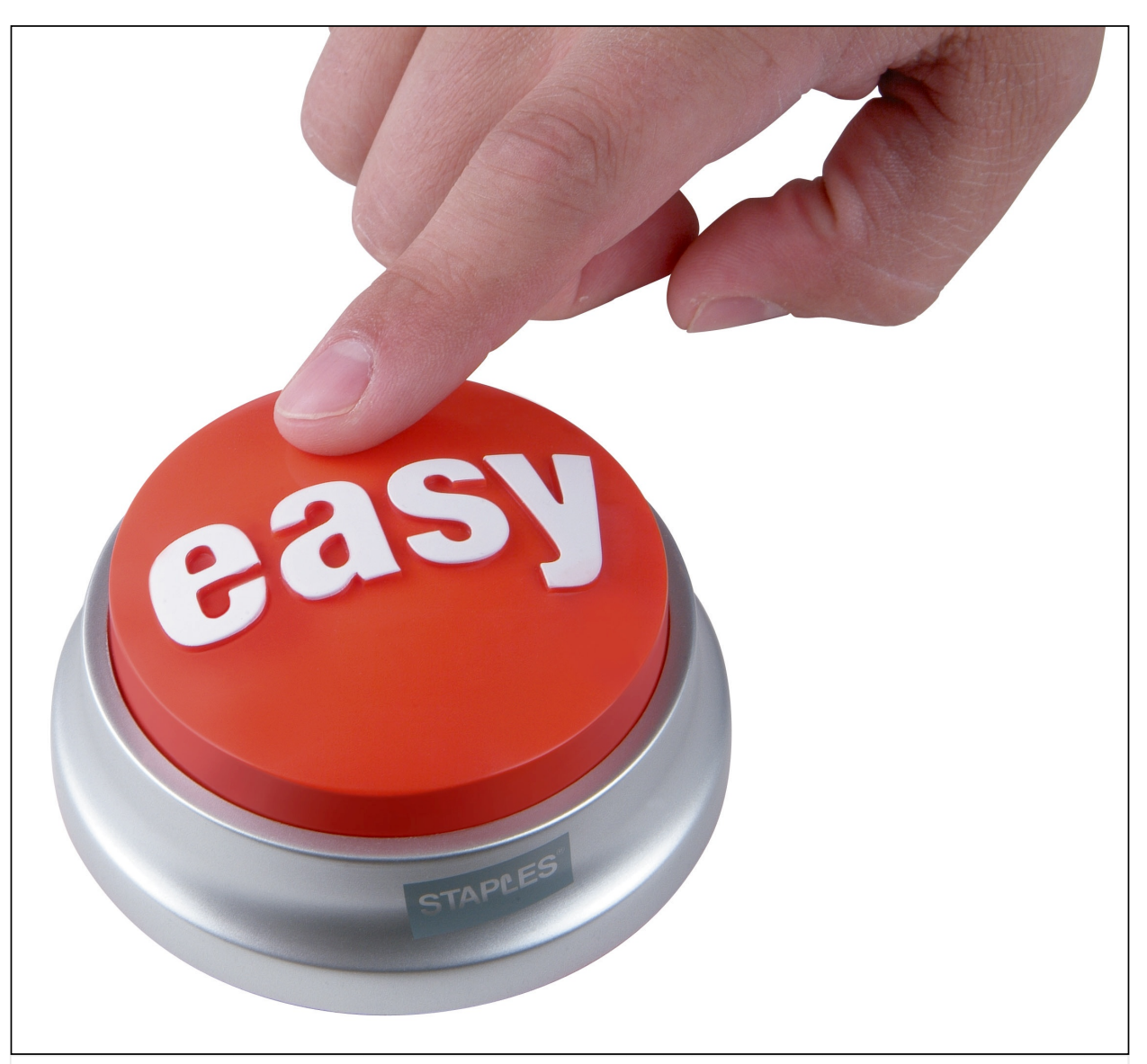

Vision

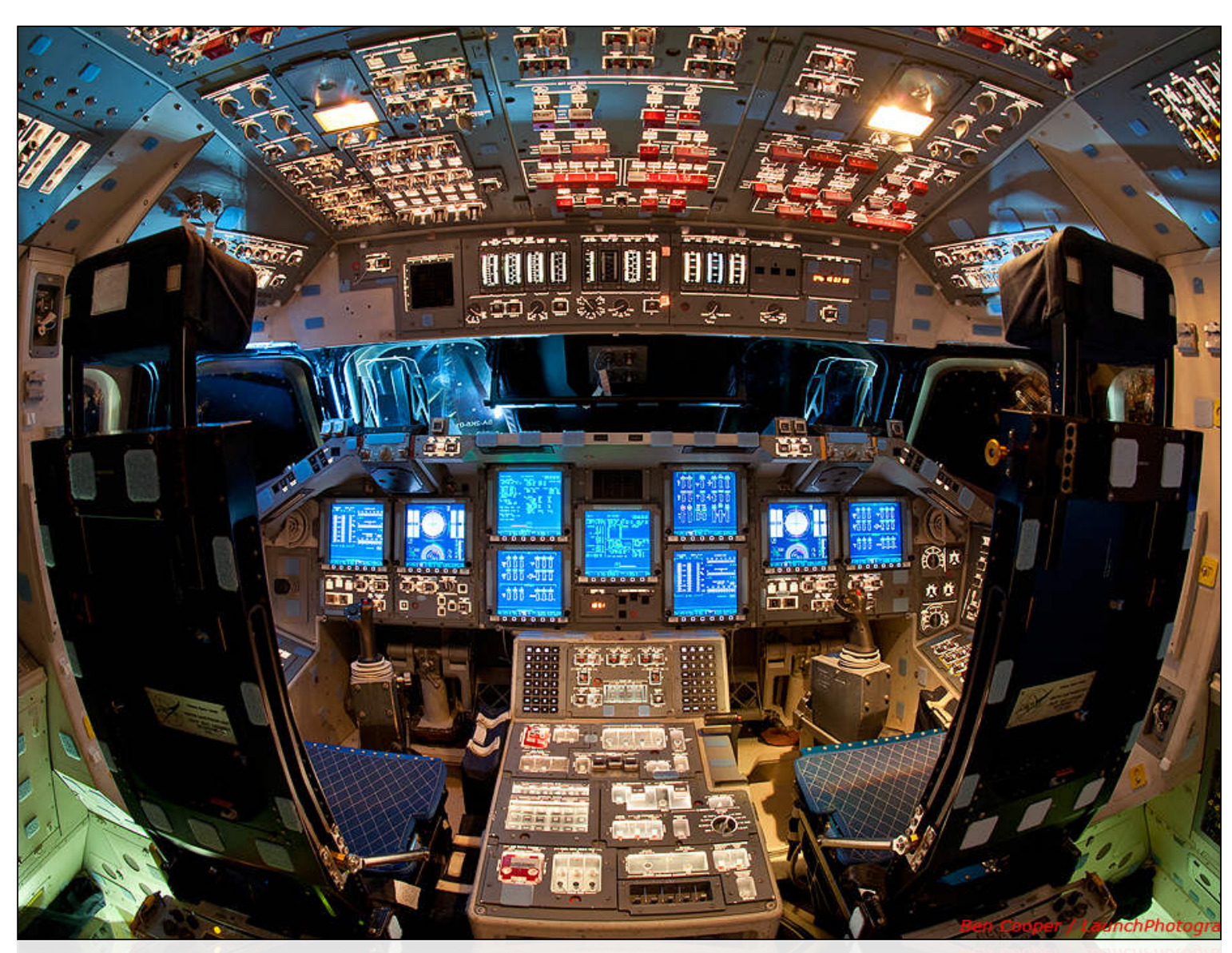

Reality 
- Clustering is one of the most basic yet most powerful and fundamental of machine learning algorithms

- But even in this simple setting, the choice of parameters are both difficult and greatly impact performance 
- Clustering is one of the most basic yet most powerful and fundamental of machine learning algorithms

- But even in this simple setting, the choice of parameters are both difficult and greatly impact performance

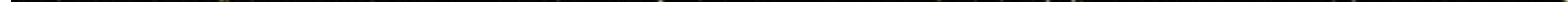


If machine learning is fundamentally a data driven science, shouldn't the use of machine learning itself follow a data driven methodology? 
INTRODUCTION

- We present Lambda Means, a meta algorithm for the newly popular clustering algorithm DP-means

- Lambda Means automatically finds DP-means' main parameter $(\lambda)$ automatically

- It finds $\lambda$ using the data itself on which the clustering is being performed 
TALK OUTLINE

- Motivation and Introduction

- Background

- Lambda Means

- Benefits of Lambda Means

- Results

- Extension to Distributed Framework 


\section{DP-MEANS}

- DP-means forms clusters of superior quality using a distance parameter $\lambda$ to ensure minimum separation between cluster centroids rather than specifying $\mathbf{k}$ in advance

- B. Kulis and M. I. Jordan (the authors of DP-means) show that this new algorithm outperforms the traditional k-means algorithm!

- The algorithm forms a new cluster when a data point is found to be more than $\lambda$ distance away from all existing cluster centroids 


\section{DIRICHLET PROCESS}

- Under an assumption that a sequence of data is drawn from a Dirichlet Process

Mixture Model, B. Kulis and M. I. Jordan (the authors of DP-means) prove that there exists a lambda value such that when used by DPmeans, the algorithm will discover the ground truth number of clusters $k$.

$\boldsymbol{\mu}_{1}, \ldots, \boldsymbol{\mu}_{k} \sim G_{0}$

$\boldsymbol{\pi} \sim \operatorname{Dir}\left(k, \boldsymbol{\pi}_{0}\right)$

$\boldsymbol{z}_{1}, \ldots, \boldsymbol{z}_{n} \sim \operatorname{Discrete}(\boldsymbol{\pi})$

$\boldsymbol{x}_{1}, \ldots, \boldsymbol{x}_{n} \sim \mathcal{N}\left(\boldsymbol{\mu}_{\boldsymbol{z}_{i}}, \sigma I\right)$

- $\mu$ corresponds to the mean of each of the clusters, drawn from some base distribution $\mathrm{GO}$, which is the prior distribution over the means

- $\pi=\left(\pi_{1}, \pi_{2} \ldots\right)$ corresponds to the vector of probabilities of being in a cluster ( $k \rightarrow$ infinity)

- $z_{i}$ is an indicator of cluster assignment

- $x_{i}$ is a data point 


\section{DP-MEANS}

- In practice, without knowing the parameters of the distribution from which the data is drawn, it is unclear how to find the appropriate value of $\lambda$ for use with DPmeans

- To solve this problem, a Farthest-first Heuristic requiring a user-provided approximation of $\mathbf{k}$ can be used

- However, it is not easy to set $\mathbf{k}$

- The choice of $\mathbf{k}$ has a marked impact on the resulting value of $\lambda$ 
TALK OUTLINE

- Motivation and Introduction

- Background

- Lambda Means

- Benefits of Lambda Means

- Results

- Extension to Distributed Framework 


\section{LAMBDA MEANS}

- As a solution for automatically finding the $\lambda$ parameter for use with DP-means, we present Lambda Means

- It finds $\lambda$ using the data itself on which the clustering is being performed

- Under an assumption that the data is generated by a Dirichlet Process Mixture Model, we formally prove that the $\lambda$ value found by Lambda Means is the same $\lambda$ used in generating the data (see Section III.D in our paper) 


\section{LAMBDA MEANS}

- The algorithm's main mechanism is to decrease $\lambda$ at each iteration, automatically terminating at the proper $\lambda$ value

- This has the effect of precipitating clusters at each iteration up to the point at which all clusters have been identified, but before the point at which true clusters are broken up into individual points 


\section{ILLUSTRATION OF EFFECT OF DECREASING $\lambda$}

Iteration: T

Lambda: Large

A large value of lambda

causes the two sets of

points to be clustered

together

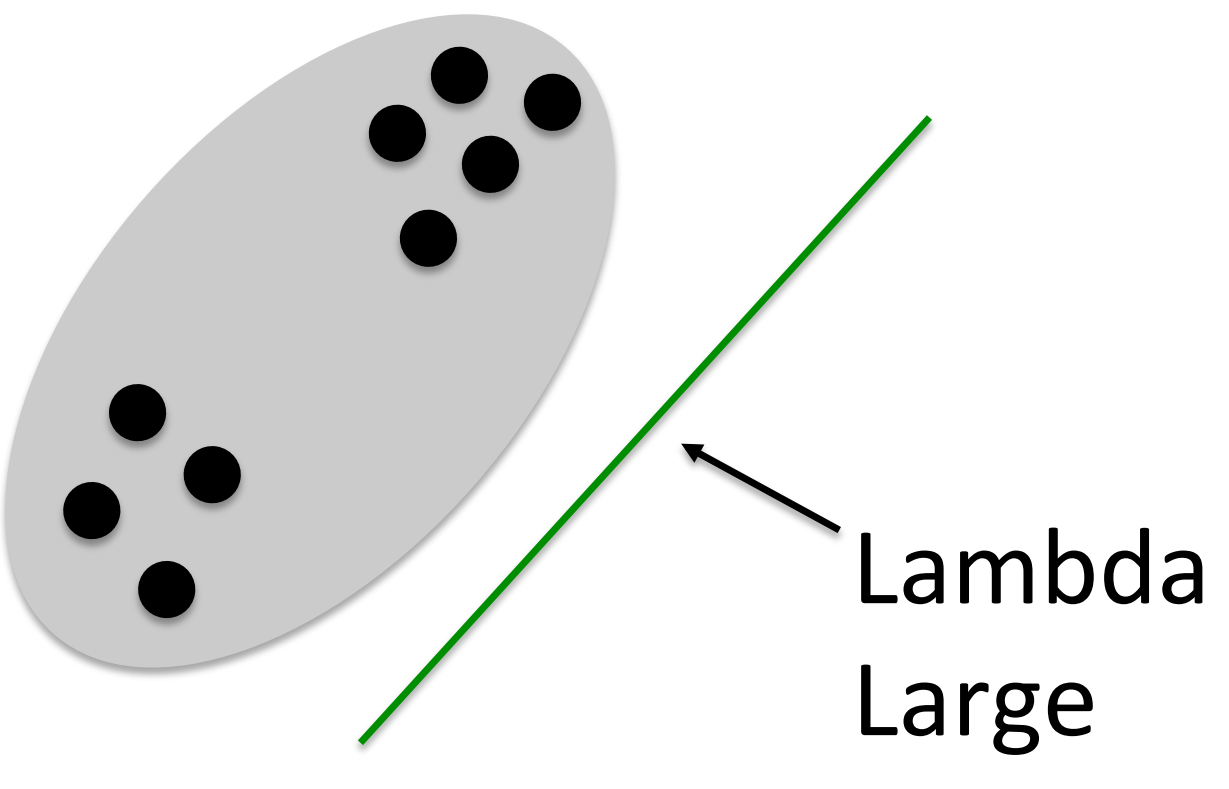

Iteration: $T+\Delta T$

Lambda: Small

A small value of

lambda causes the two

sets of points to be

clustered separately

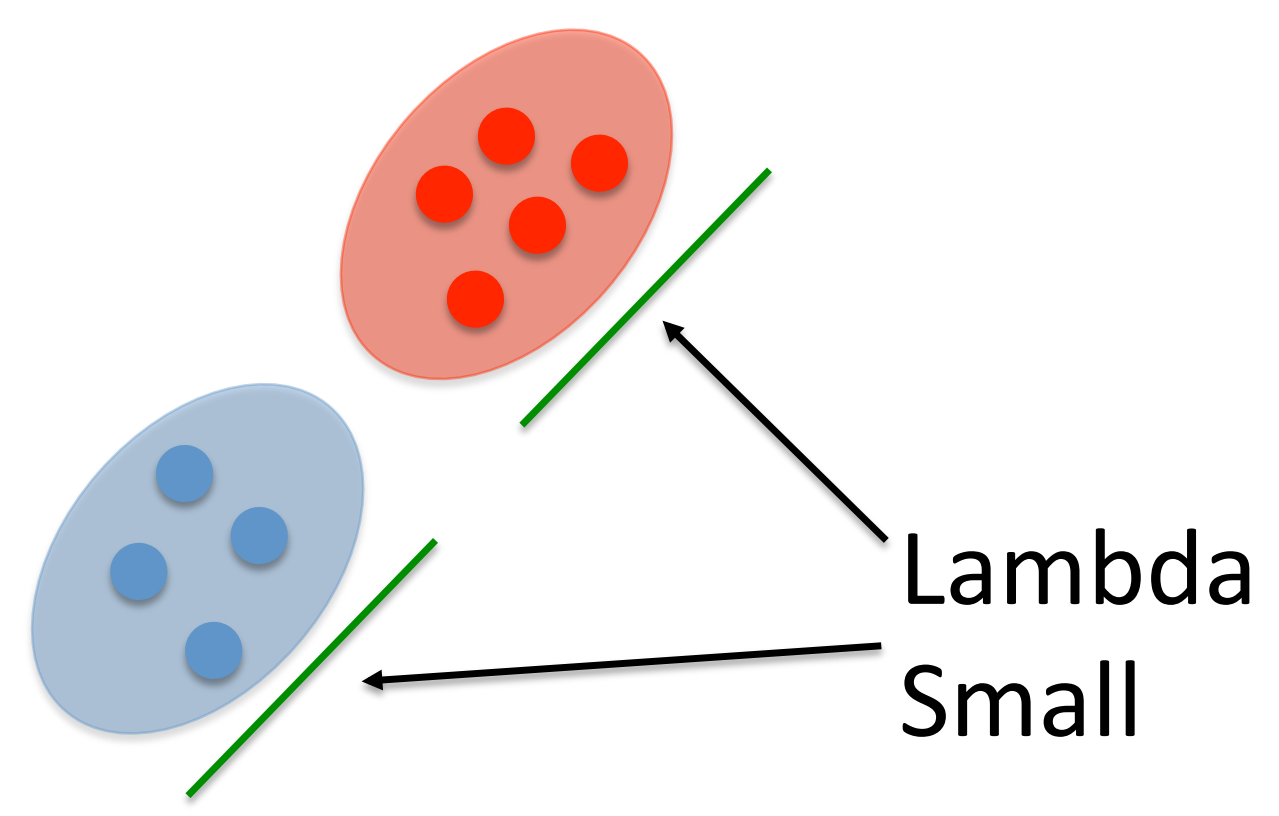




\section{ILLUSTRATION OF EFFECT OF DECREASING $\lambda$}
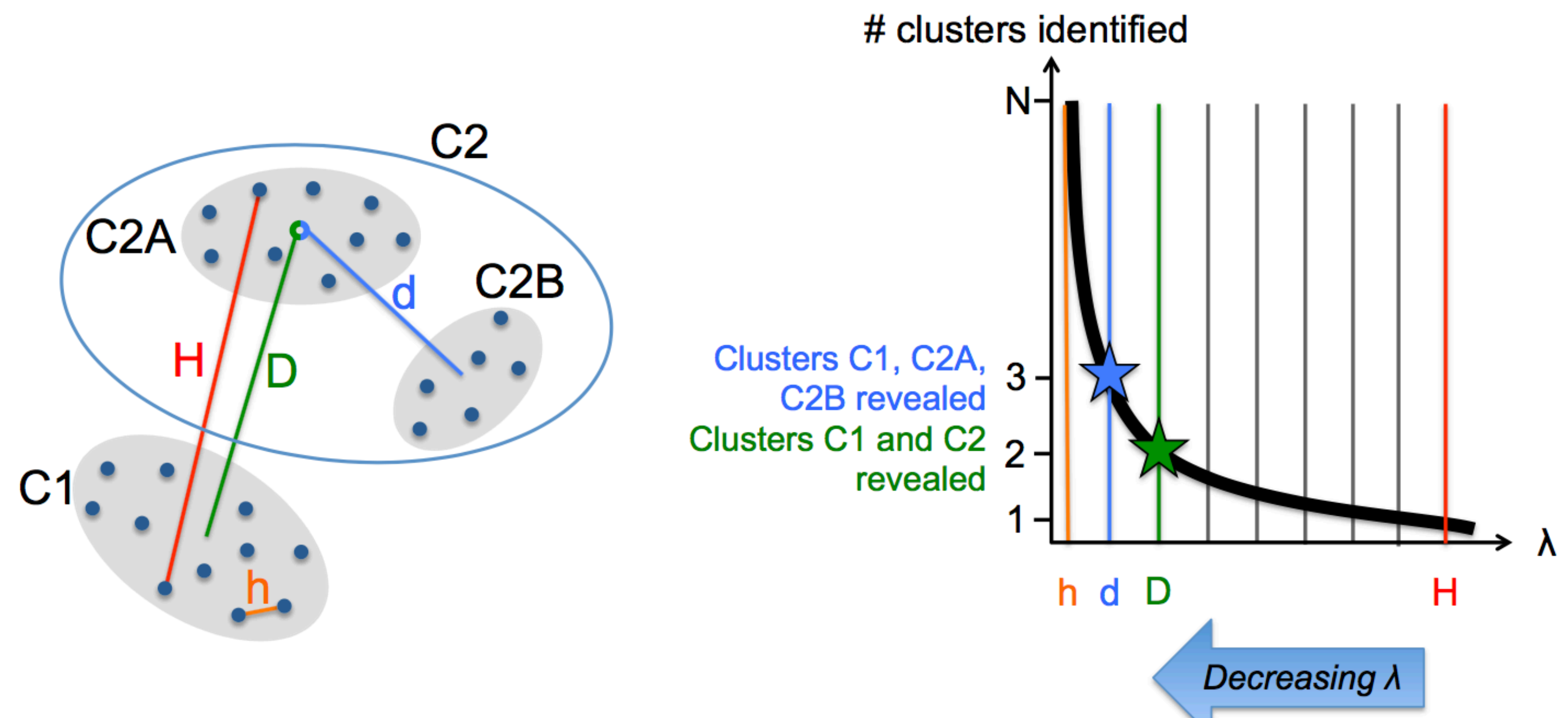

$\mathrm{D}, \mathrm{d}$ : maximum and minimum distance between cluster centroids $\mathrm{H}$, h: maximum and minimum distance between data points $N$ : total \# of data points 


\section{LAMBDA MEANS}

- Note that a naive implementation would generate the entire curve and then search for the elbow

- Lambda Means replaces the need for this exhaustive search for the elbow of the curve

- The algorithm uses the cumulative number of clusters formed as a signaling

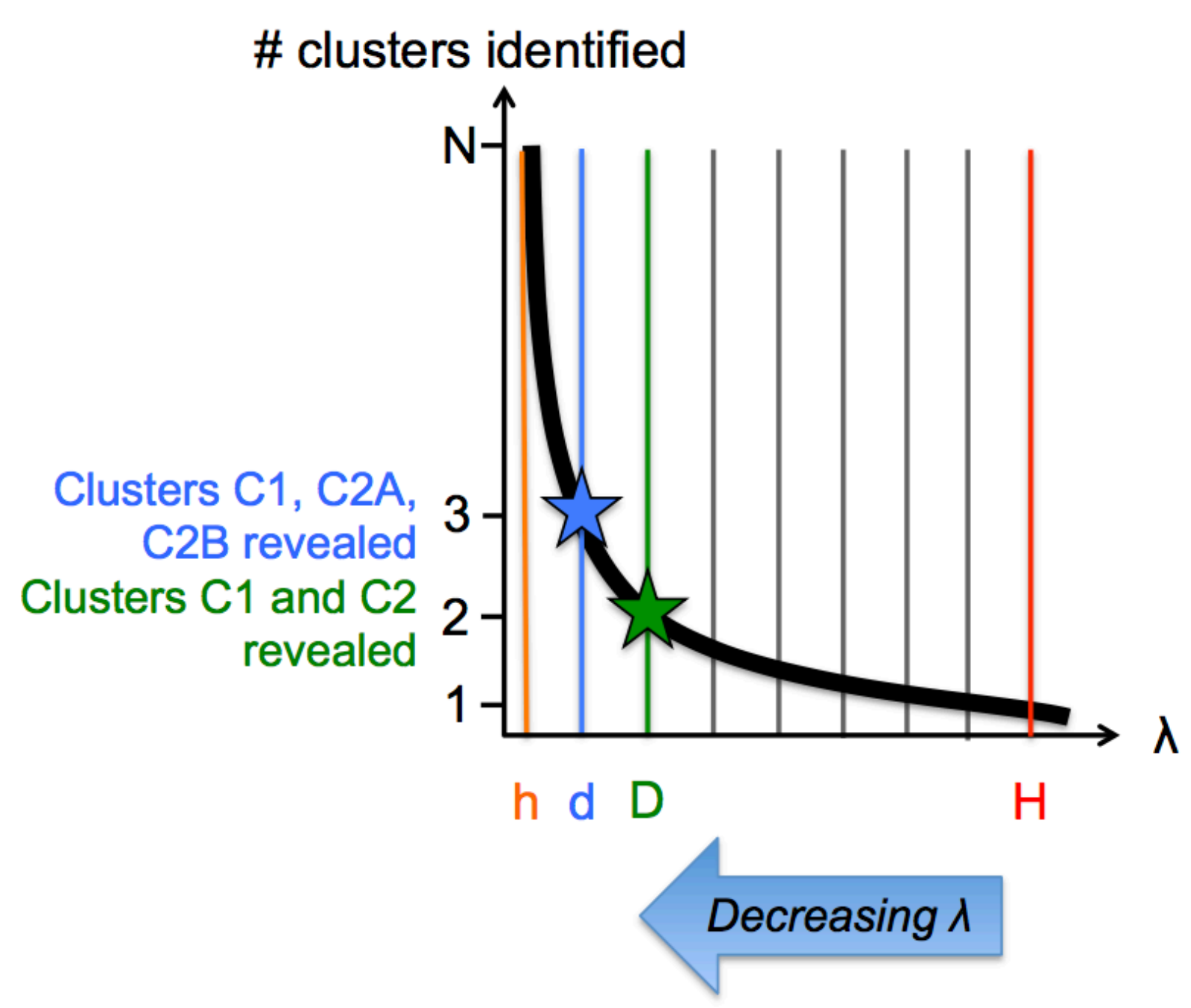
mechanism, continuing to iterate with smaller values of $\lambda$ until the stopping criteria is met 
TALK OUTLINE

- Motivation and Introduction

- Background

- Lambda Means

- Benefits of Lambda Means

- Results

- Extension to Distributed Framework 
- Lambda means is more robust then using a Farthestfirst Heuristic, which requires a user-defined $\mathbf{k}$

- Reason 1: Setting this $\mathbf{k}$ can be very difficult

- Reason 2: If the initial approximation to $k$ is wrong, it negatively affects finding the correct $\lambda$ 
- To show the effect of an incorrect $\mathbf{k}$, we generate a dataset and then use the Farthestfirst Heuristic with a number of different values of $k$ to derive $\lambda$

- We find that $\lambda$ varies greatly based on the

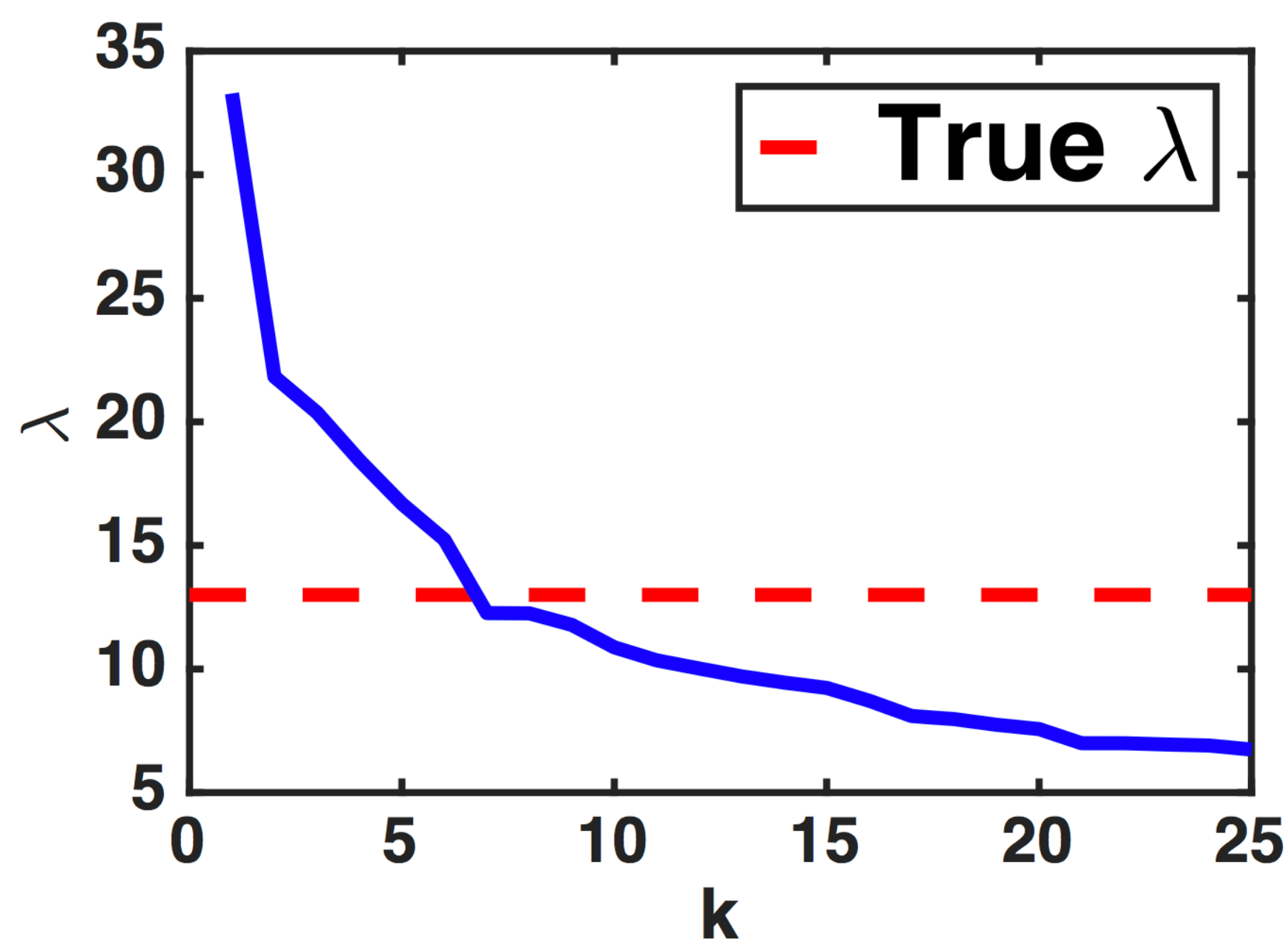
initial k used 


\section{BENEFITS}

- The drawbacks of the farthest-first heuristic are clear:

- The method is brittle to small changes in the approximation of $\mathbf{k}$

- The method has a large impact on the derived value of $\lambda$ as well as potentially on the resulting cluster quality

- In contrast, Lambda Means automatically finds the $\lambda$ value without an initial approximation for $\mathbf{k}$ 


\section{TALK OUTLINE}

- Motivation and Introduction

- Background

- Lambda Means

- Benefits of Lambda Means

- Results

- Extension to Distributed Framework 
RESULTS

- We provide experimental evaluation of $\lambda$-means on both synthetic and real world data

- For synthetic data, we generate data with different values of inter-cluster variance $\boldsymbol{\rho}$ and the intra-cluster variance $\boldsymbol{\sigma}$

- For real-world data, we use the MNIST hand written digit dataset 


\section{RESULTS}

- This figure shows that for synthetic data with a high value of $\boldsymbol{\rho} / \boldsymbol{\sigma}$, Lambda Means is able to automatically find the $\lambda$ value that maximizes AMI and NMI scores

- NMI measures the amount of mutual information normalizing for number of clusters, and AMI measures the amount of mutual information accounting for chance

- We can also judge Lambda Means by its ability to identify the correct number of clusters, which it does (as shown by the blue line)

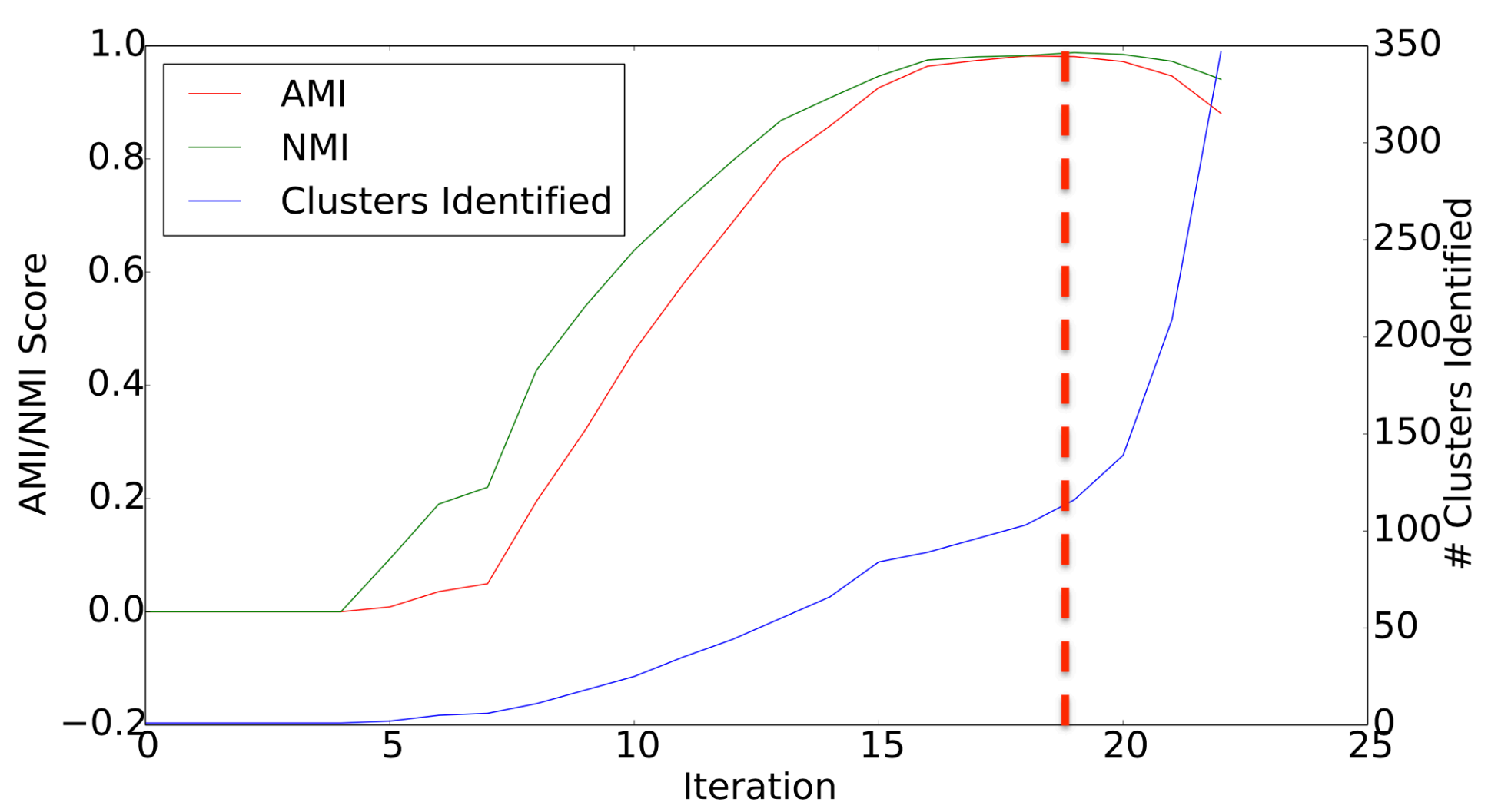


RESULTS

- We now compare the AMI and NMI scores for Lambda Means and DP-means in Table I for additional values of $\rho / \sigma$, as well as for the MNIST dataset

- Lambda Means outperforms DP-means where $\lambda$ is set via the Farthest-first heuristic

\begin{tabular}{|c|c|c|c|c|c|c|}
\cline { 2 - 7 } \multicolumn{1}{c|}{} & \multicolumn{9}{c|}{ Dataset } \\
\cline { 2 - 7 } \multicolumn{1}{c|}{} & \multicolumn{2}{c|}{ Syn. $\frac{\rho}{\sigma}=15$} & \multicolumn{2}{c|}{ Syn. $\frac{\rho}{\sigma}=5$} & \multicolumn{2}{c|}{ MNIST } \\
\hline Algorithm & AMI & NMI & AMI & NMI & AMI & NMI \\
\hline$\lambda$-means & 0.97 & 0.98 & 0.77 & 0.82 & 0.43 & 0.53 \\
\hline DP-means & 0.87 & 0.92 & 0.52 & 0.78 & 0.32 & 0.38 \\
\hline
\end{tabular}




\section{TALK OUTLINE}

- Motivation and Introduction

- Background

- Lambda Means

- Benefits of Lambda Means

- Results

- Extension to Distributed Framework 


\section{DISTRIBUTED RESULTS}

- Lambda Means easily extends to the distributed framework under the optimistic concurrency control framework

- We achieve within a factor of two away from a perfect speed-up in both the multicore and multi-processor distributed settings

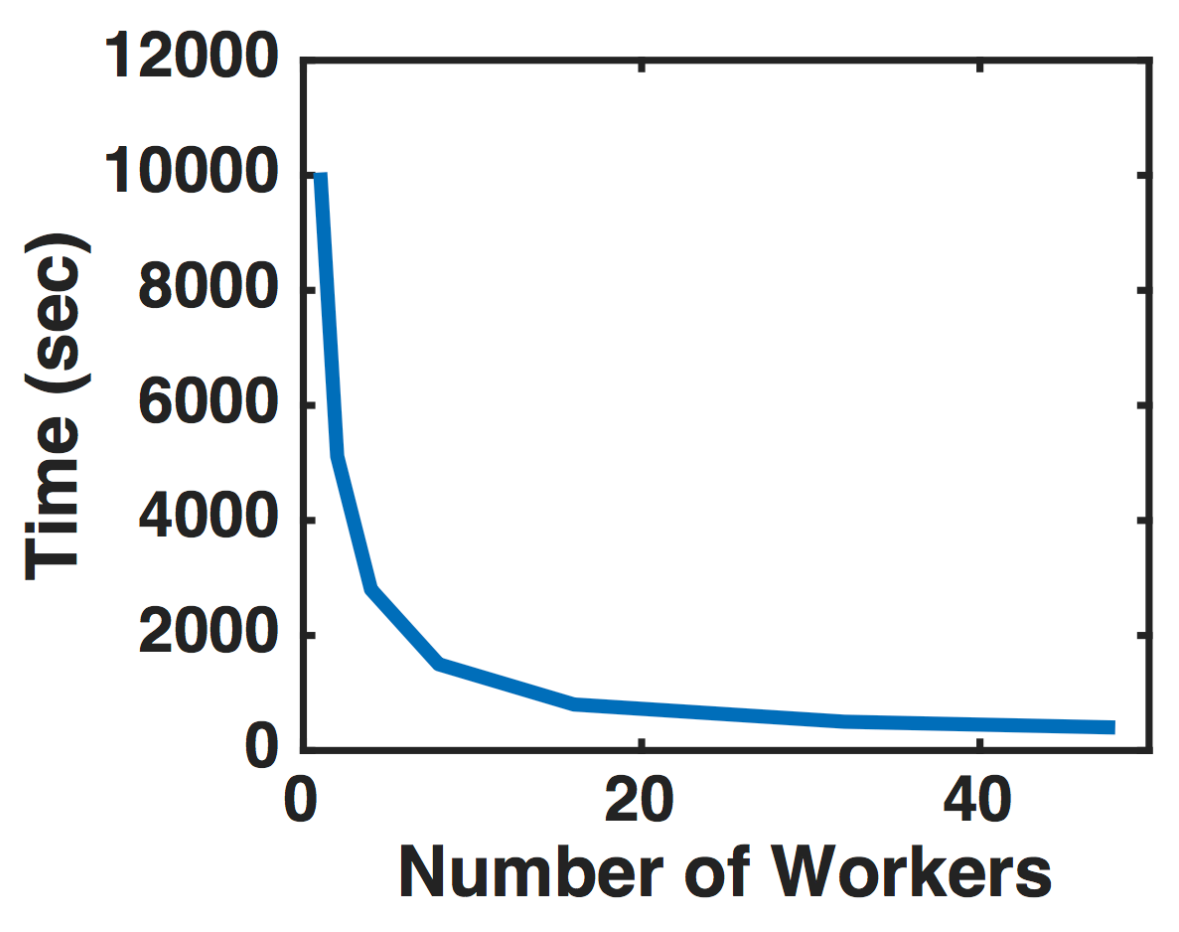

(a) Distributed cluster in the cloud

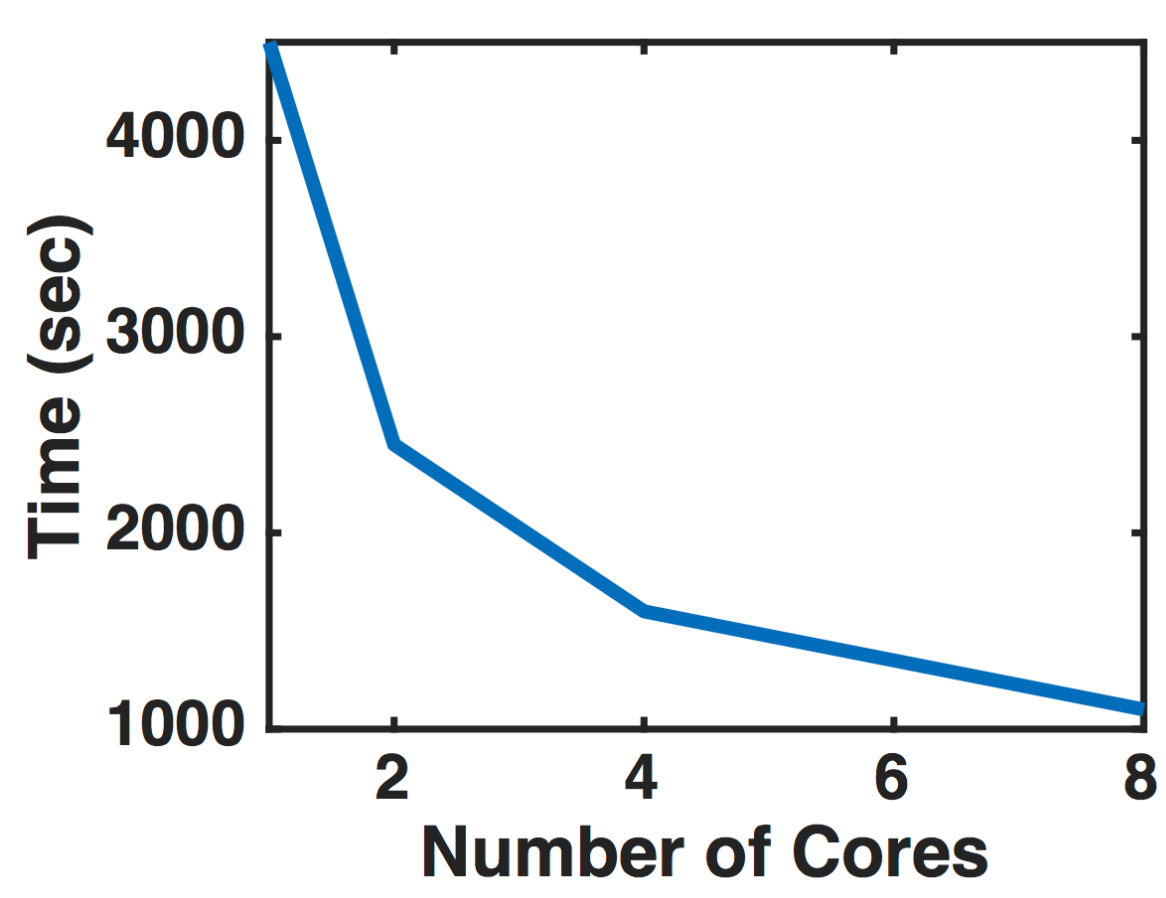

(b) Multicore 


\section{THANK YOU}

MARCUS COMITER, MIRIAM CHA, HT KUNG, SURAT TEERAPITTAYANON HARVARD UNIVERSITY 